\title{
Informational Mechanisms Created While Biological Evolution: Deductive 'Construing' - from Elementary Cells to Intuition and Harmony
}

\author{
Vladimir M Petrov* \\ Department of Biostatistics, Russia
}

*Corresponding author: Vladimir M Petrov, Department of Biostatistics, Russia.

Received Date: July 09, 2019

Published Date: July 16, 2019

\begin{abstract}
In the framework of the systemic-informational approach, interactions between sets of 'computational blocks' (e.g., neurons or other units) are subdued to optimization. The resulting multi-level hierarchy responds to statistical regularities inherent in biological, social, and cultural systems, first of all the 'power law' (hyperbolic distribution) and 'regression to the mean.' Extrapolating a set of partial models dealing with information processing and formed while biological evolution, we come to the need for additional 'tools' named 'emotion' and 'intuition.' Their bases should be 'harmony' in co-existence of different levels of the hierarchical structure deduced. Some extremely low-probable events occur needed and possible, permitting to speak of approaching to 'general scientific theory of miracles.'
\end{abstract}

Keywords: Information; Entropy; Psychology; Statistics; Optimality; Regression; Power Law; Neurons; Intuition; Extrapolation; Correlation; Resource; Hierarchy; Witches; Miracles; Forecasting

\section{Introduction}

Oh, I remember that terrible time of war - the epoch of total hunger: at that period, I was a chief of a mincing-machine. So, each night I came to the hop, to dance, and simply poked my finger at a couple of sluts: you, you, and you. And each of them went to my home - because of a sound bite of pork! From stories narrated by an old Soviet man who survived the Second World War and hopes to survive the Third one. The paper deals with events, belonging to various fields of knowledge: psychology, general system theory, sociology, cultural studies, etc. That is why we resorted to the help of rather specific approach (not widespread in each of these fields): the so-called 'systemic-informational analysis.' It eliminates the peculiarities inherent to the fields mentioned, permitting to embrace them - and to enter the world of 'new mental reality.' (Earlier, this approach demonstrated its effectiveness when integrating various spheres: linguistics, ethics, general history, international relations, psychology, art studies, and so forth; see, e.g., [1-7]. Some of the events to be analyzed in this text, are partial and seem to be very significant neither for our life, nor science, - but certain events (e.g., contemporary state of international relations and possible menace of the world war) are very important! And all of them are united by high impact of intuitive (and/or subconscious) processes.

Lyrical digression number one ('autobiographical'). I remember the situation which took place on December 31, 1999, - when we were going to celebrate the New Year, - meaning our small circle of several creative persons (composers, writers, painters, biologists, statisticians, and physicists). All around us was awful, absolute disorder was everywhere: political chaos and voluntarism were accompanied with a decay in economics, mental life and arts, etc. Failed our hopes concerning perspectives of 'democratic development' of Russia, as well as hopes for support of Western Europe, and other high spirits and dreams. We were disappointed by Russian President Boris YELTSYN and his reforms. HALF AN HOUR BEFORE THE MIDNIGHT, I was asked to propose a toast to the future. And SUDDENLY the full IMAGE OF THE END OF THE NEXT YEAR - appeared in my sight: the picture was still awful, but it didn't contain President Yeltsyn! So, in my toast I forecasted the absence of Yeltsyn - in the role of President - at the end of 2000: this person seemed me to be INCOMPATIBLE with the entire picture! 
At that moment, nobody believed my INTUITION AND PROGNOSIS, - but exactly on December 31, 2000 (i.e., precisely one year later), just before the midnight - we were again together - and knew about RADICALCHANGE of the leader of our country: suddenly Vladimir PUTIN became our chief. At that moment, everybody remembered my forecast, which occurred a MIRACLE! Since that time, I started to pay more attention to 'harmony' of the forecasted image of the future, i.e., co-ordination of its various features (their statistical 'fitness' to each other), organized by our intuition. [I have no comments concerning Vladimir Putin personally. Moreover, till now his personality remains rather vague and mysterious for me as well as for some of my colleagues and friends. First time I heard this name - as a future leader of Russia - from one of my American students, when delivering lectures at the University of California, Santa Cruz, in August 2000. (So, at that time, one of my students which afterwards became my friend, - was more informed about Russian current events, than me!) However, the matter is not in the detailed information about some features of personages involved, but in their 'harmonious fitness' for the entire socio-psychological 'climate' of time: Yeltsyn didn't respond to any 'current harmony' - except the traditional image of Russian Hercules, drinking vodka every day.]

However, farewell to lyrics: it is time to turn to some results obtained in the framework of the systemic-informational approach. So, adieux till next digression!

\section{Evolution: from elementary primary cells - to multi- level hierarchical structures}

Our approach proceeds from rather famous statistical "principle of maximization of mutual information" between the system studied - and its environment. [The system may be a worm, a human being, forest, species, language, society, kind of art, and so forth.] We will reproduce the logical line of deductive 'construing' certain psychological and cultural structures, which would be consequences of the above maximization; more detailed description of our 'construing' see in: [1,4]. As it is known, the mutual information between two variables $\mathrm{x}$ and $\mathrm{y}$ can be defined [8] through the symmetrical expression:

$$
\mathrm{I}(\mathrm{x}, \mathrm{y})=\log [\mathrm{p}(\mathrm{x}, \mathrm{y}) / \mathrm{p}(\mathrm{x}) \mathrm{p}(\mathrm{y})],
$$

where $\mathrm{p}(\mathrm{x}), \mathrm{p}(\mathrm{y})$ are probabilities of the values $\mathrm{x}$ and $\mathrm{y}$; and $\mathrm{p}(\mathrm{x}, \mathrm{y})$ is the joint probability of the combination of $\mathrm{x}$ and $\mathrm{y}$. When the parameters $\mathrm{x}$ and $\mathrm{y}$ relate to the environment and the given system respectively, mutual information characterizes the "fitness" of the system for the environment [2].

We would remind of the principal condition of such optimization: a certain value - the so-called Lagrangian - is to be maximal:

$$
\mathrm{L}(\mathrm{X}, \mathrm{Y})=\mathrm{H}(\mathrm{Y})-\mathrm{H}(\mathrm{Y} / \mathrm{X})-\beta \mathrm{R}(\mathrm{X}, \mathrm{Y}) \rightarrow \max ,
$$

where $H(Y)$ is the entropy of the system's states, $H(Y / X)$ the entropy of the system's errors in responses, $\mathrm{R}(\mathrm{X}, \mathrm{Y})$ the average resource expense for the system's states $\mathrm{Y}$ and the environmental states $X$, and $\beta$ the indicator of the deficit of the resource $(\beta=0$ when the system possesses unlimited resource, and $\beta=1$ when strong resource deficit). The role of resource can be played by different 'substrates': in economics it might be money, in mechanics - energy, in chemistry - substance, in biology - the amount of various species, in sociology and cultural studies - the number of creative persons in the society, etc. As far as the sum of three items is to be maximized, we have three fundamental tendencies inherent to the system's behavior:

I. Expansion - the aspiration to increase the number and the variety of the environmental states in which the system can exist. This is possible due to growth of the variety of the system's responses H (Y). Such tendency is often named 'search behavior.'

II. Idealization - the aspiration to increase the 'exactness' of the system's responses, i.e., to decrease the entropy of the system's behavioral errors $\mathrm{H}(\mathrm{Y} / \mathrm{X})$. This tendency is sometimes called 'conservative inclination.'

III. Economy of resources. This item contains two multiplied constituents. Hence, it can be expressed either in the choice of the system's states responding to minimal average resource expense $\mathrm{R}(\mathrm{X}, \mathrm{Y})$, or in the aspiration to decrease the deficit of resource $\beta$, i.e., to increase resource supply.

The above set of tendencies - how does it work in relation to the structure of the system considered? Of course, usually these three tendencies are tightly connected, interwoven. However, only the first tendency (A) and the second one (B) deal immediately with the system's states, whereas the third tendency (C) is capable of influencing upon these states only indirectly, through the resource requirements. [The role of the resource tendency is similar to the functions of book-keeping in a large contemporary company: usually the main function of the book-keeping is to inform the head of the company about possible financial limitations when choosing the direction of further development of the company, out of possible versions, these versions themselves being derived by appropriate company's departments.] That is why it seems reasonable to proceed from the first and the second tendencies - to come to some conclusions concerning the system's parameters. We shall 'construe' the evolutionary trajectory of informational structures, as if a certain 'engineer' would choose the most 'advantageous' decisions, inventing new 'tools' at each moment of the evolution, in accordance with the above three fundamental tendencies. [Some potentialities may be lost when such 'construing,' which are not subdued to the 'concept of gradual changes,' - but these are not inherent to the evolution.]

Our starting point will be the evident fact of nowadays existence of various rather perfect, 'well developed' systems: the forest, the human being, language, some kinds of art, etc. Meanwhile, once upon a time (many millions years ago), there was no ordering (or almost no ordering) in the Universe: everywhere disordered elements (atoms and/or molecules) were simply mixed, without 
(or almost without) any hierarchy, structure, etc. Two principal ways are possible to construe the trajectory between these 'extreme states' - for any system (a path from initial disordering - to nowadays highly structured state). The first way deals with certain fixed object, though it needs 'polishing' some of its features - gradually coming to more and more perfect structure. Example of such system is a stone at the beach: it experiences some internal (structural) transformations; as well, the stone is polished by the waves. But in principle, while the evolution, we deal with the situation of 'object's identity': it is the same object (the given stone).

The second way is quite opposite: the system consists of elements which are constantly replacing each other, as if they belonged to different generations; so the 'identity' of the system relates not to objects themselves - but to definite invariants over temporal changes. There are many examples of such systems relating to biology, demography, cultural studies, and so on. For instance, the flux of generations within the biological species, is constantly changing, so the 'identity' of the given species - as a system - can be ascribed only to the invariant, i.e., the entire flux, but not to its separate elements. Our further consideration will follow the logic of this second way, which seems to be more effective. Let's turn to the above two principal systemic tendencies (A, B). Which of them should be prevailing at the early stages of the evolution of any system? - Of course, when the system is rather 'weak,' it would be desirable to appeal to both tendencies! But out of these two - exactly that tendency seems to be more 'useful, which deals immediately with inter-element processes, and first of all, with their increasing scales (growing sizes of functioning). So, we should start from the first tendency - expansion.

The system' activity can be treated as 'trials' to combine various elements which are present in the environment. Out of theses combinations, it is possible to single out those ones which form 'steady alliances,' i.e., they occur so tightly connected with each other, - that the 'fruit' of their combining would be stable, resisting to most further combining which would require replacing one of the constituents. [Thus, in case of such elements as atoms, their combining can result in formation of molecules; e.g., two atoms of hydrogen combined with an atom of oxygen, produce the molecule $\mathrm{H}_{2} \mathrm{O}$; here the criterion of the alliance stability, is to have 8 electrons at the outward orbit of each atom combined.]

In turn, out of such steady combinations, certain ones - the most 'strong,' 'mighty' - should be singled out, which satisfy the conditions named 'autocatalytic.' (Because the most obvious examples of such situations relate to the sphere of chemistry.) Here the heart of the matter is such a specific (partial) case of elements' interactions, when they support (and even strengthen) the process of combining - through the 'identity' of one of its constituents with final result ('fruit') of combining. Due to this, the "positive feedback" takes place, capable of producing 'self-organization' within a definite fragment of the space. For instance, when reaction between substances $\mathrm{P}$ and Q:

$$
P+2 Q \rightarrow 3 Q,
$$

one molecule of $\mathrm{P}$ is transformed - in 'catalytic' presence of molecules Q - into one (additional) molecule of substance Q. Such autocatalytic reaction is usually depicted graphically in the form of a loop (Figure 1).

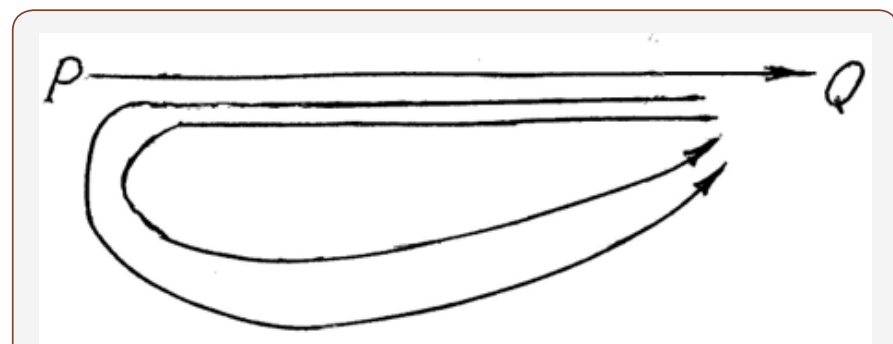

Figure 1: Scheme of the loop characterizing the logic of autocatalytic reaction.

From the methodological point of view, we deal with the phenomenon of "reflexion" - in a genuine scientific sense of this term: "as the transformation of the conditions of the control ('means') into the aim of the control ('end'), as the transfer of the control from the effect to the cause. This looks like a 'reverse motion' along the causal bonds from the end to the beginning" [2]. The phenomenon of reflexion should display itself (and it did really display itself) in various fields and on various stages of the evolutionary process. Moreover, further this phenomenon will occur the most important principle of the evolution. "This principle provides the qualitative jump in the development of the system, the transition of life to a new level of organization. There are many 'thresholds' and 'jumps' on the way of evolution. One of them is the jump from the non-living matter to living matter, when Nature had to build very large and complicated molecular structures (polypeptides and polynucleotides) to provide self-reproduction. The other well-known jump was the jump from the animal to man, the origin of human consciousness.

Many investigators felt special mechanisms had to exist to overcome such thresholds. Hypercycle proposed by M. Eigen [9] is an example of such a mechanism. Reflexion is the general principle of the organization of such mechanisms. Reflexion as a principle is inherent to the nature of the informational interaction as such: a control system can control itself, can be an object of self-control. There is a variety of mechanisms of control on different levels of organization, but the principle remains the same. Reflexion allows a new control level to be 'built up' on top of the old one $<\ldots>$ One of the most important consequences of reflexion was the emergence of the hierarchical organization of the control processes in the living organism. A new level of hierarchy arises when any control process becomes the object (the 'end') of another control process. It opens the possibility for the directed perfection and optimization of the first process. One may say that all progress in the evolution of life, is based on the hierarchical organization and optimization of the control systems and processes by means of reflexion. The result of such development was the emergence of multi-level hierarchical control structures. In these structures information is transmitted from the lower level to a higher one, where it is evaluated, selected, and processed. On the other hand, the higher level transmits to the 
lower one controlling influences, which determine the work of this level" [2]. The scheme of such hierarchical structure is shown by Figure 2.

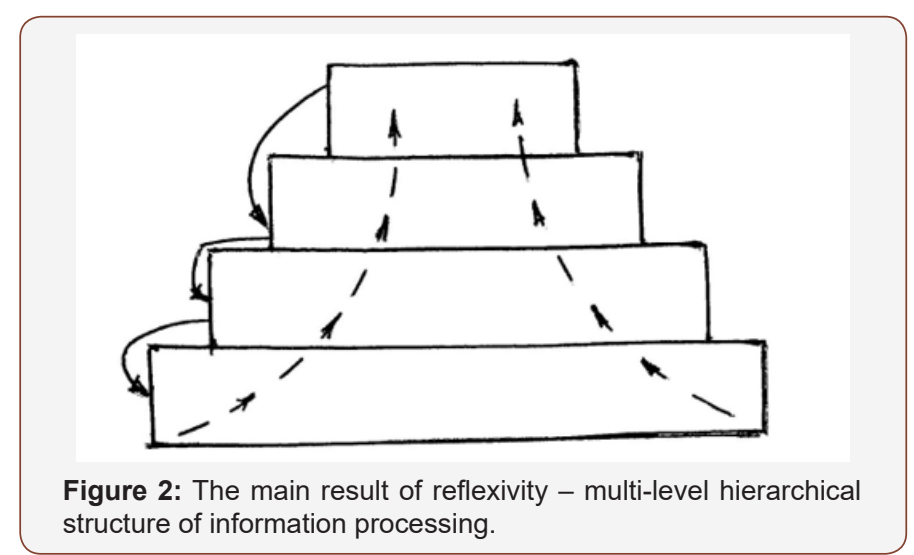

Reflexive structures penetrate all the stages of the evolution: from its very beginning - to contemporary one. Meanwhile, evidently to materialize such structures (filled by reflexive 'interior'), it is desirable to defend them from outer destroying interactions - by means of something like a border. Perhaps, here our 'construing' should resort to the help of the second fundamental tendency (B idealization)? - Yes, it occurs to be reasonable, because exactly the border is the best tool capable of realizing minimal errors when the system's interacts with its environment. This tool is subdued to two kinds of requirements:

I. The border should not be 'absolutely deaf' - it should provide certain transfer both of energy (needed for the system's functioning) and information (at least concerning changes in the environment); otherwise, the system wouldn't exist;

II. The border should function as a certain 'instrument for selection,' e.g., providing transmission of some signals (or substances), and prohibiting transfer of some others.

A brilliant example of satisfying both requirements is well known: such evolutionary device as the membrane (see in detail [4]). This rather old 'tool for selection' permits to distinct between different substrates and apply different criteria for their transfer. In other words, chaos and disordering in the surroundings, are opposed by order and organization behind the 'fence,' i.e., low entropy. Nevertheless, in spite of such isolation, the membrane gives the opportunity to interact with the environment: to receive both energy and information. Due to this evolutionary 'invention,' the living matter could create its own 'dwelling places,' certain 'islands of autonomy' appeared, where the entropy started decreasing. This device had many faces (echoes) at various phases of the evolution, in the form of the skin, hairs, burrow, and so forth. On the territory of such 'islands,' the living matter could show growing selforganization, in particular of that hierarchical kind which has been considered above. So, these islands provide the space for reflexion (the potentialities of which are great, which was shown earlier). So, hierarchical structures can become the reality!

Hypothetical process of forming such hierarchical structures - from the 'soup' of elementary units (e.g., neurons), realizing informational interactions with each other, - was considered earlier - see, e.g., [4]. In the framework of one of contemporary models, a set of elementary units is supposed to be engaged in information processing, each unit receiving information from 2 or 3 outside units - and transmitting appropriate impulses (the results of processing) to another unit. [Preference for 2 or 3 'input channels' was deduced theoretically, irrespective of concrete 'materializing' in information carriers, proceeding from the 'theory of non-motivated classifications' by Sukhotin [10]- see also below.] Due to such aggregating, the number of inter-unit channels can be diminished either $2 \cdot 2=4$ times or $3 \cdot 3=9$ times. Hence, we came to nothing else than materialized next level of the informational hierarchy which has been considered earlier (Figure 2) - as the main result of reflexion! So, our ascending path: from disordered chaos - towards highly ordered hierarchical structures - is, in principle, provided with necessary 'principal equipment.'

Lyrical digression number two (methodological). Now we have actually realized a 'jump' from micro-level dealing with cellular processes (and close ones) - to macro-level phenomena possessing rather large scales, which are inherent to social and cultural reality. [We see a lot of such 'jumps' between the realms of Non-Living Matter - and Living one!] In order to vivify our narration, I propose to mention another 'jump' - observed by me personally, in the Gobi desert of 1980's, when I was appointed a chief of joint SovietMongolian sociological expedition, the task of which was to study the mentality and style of life of Mongolian population (in order to compile the 'Long-term plan of cultural development of Mongolian People's Republic'). At that time everywhere, in all Mongolian settlements, I saw the most widespread placard - bright, proud, and impressive: 'Capitalizmug Asgulch!' (something like 'Jumping over Capitalism!'): a brave horseman symbolizing this country, was depicted in a position after having got over a giant precipice (symbolizing capitalistic stage of development, which wouldn't be obligatory, according to local Marxist 'theoreticians'). In reality, I saw quite awful consequences of a failure of such 'jump-like way towards socialism': the trajectory occurred not prepared by due intermediate steps, especially concerning mental life of Mongolian population. Nevertheless, in our given discourse, we will hopefully escape such misunderstanding - as far as our narration would deal mainly with 'key points' of the evolution.

\section{In the world of partial models: two 'eternal' statistical regularities - accompanied with their dynamic potentialities and menaces}

In the framework of the systemic-informational approach, and some close approaches - more than 30 partial models have been derived for various concrete situations (kinds of objects and/or kinds of behavior). Some of them are ready for practical applications (and these models were really used in appropriate investigations - biological, psychological, or sociological), though some are half-ready, needing further elaboration. But before our 'review of informational models' responding to various 'inventions' created while evolution (both biological and cultural), we should shortly consider certain statistical regularities which may occur 
substantial for dynamics of multi-element, multi-generation systems similar to our biological, social and cultural ones. First of all, the very changeability of any system - why is it possible? And which statistical conditions are capable of reinforcing the dynamics?

Evidently, the best way to clear up the problem of conditions, is to deduce possible distribution(s) of the system's states proceeding from the above mentioned 'principle of the information maximum' - and to find in the model construed, the 'dwelling place' for conditions in question. Recently an attempt of such kind was undertaken (see [1,7]), and one of its results occurred to be well known 'Power Law' - which is applied in biology, social and cultural studies, etc., under names of Zipf, Pareto, Lotka, Mandelbrot, and others (see, e.g., $[11,12]$. However, the 'zest' of this finding was in that now it became clear the role of conditions of the elements' functioning - in order to come to such distribution: simply it is needed that future behavior of each element would be based on its previous behavior - following the so-called Biblical "Mathew's principle" - "success generates new success." [For instance, if a researcher $\mathrm{P}$ wrote during last year 10 articles, and researcher $\mathrm{Q}$ wrote 2 articles, - then probably next year, researcher P will write several times more articles than researcher Q.]

The Power Law is 'the optimal distribution of the system's states from the point of view of the entropy maximization. <...> It describes a degree of diversity of distribution of the system's states caused by the resource restrictions' [7]. This law possesses several forms (representations). Thus, let's consider a certain system e.g., a population producing certain kind of goods. Resorting to the help of ranking, i.e., ascribing rank $r=1$ to the element producing maximal amount of goods, $r=2$ to the next producing element, and so on, - we obtain for the element of the r-th rank, the intensity of its activity:

$$
\mathrm{I}_{\mathrm{r}}=\mathrm{I}_{1} \mathrm{r}^{-\alpha} \text {, }
$$

$I_{1}$ being the intensity of the first element, coefficient $\alpha$ characterizing the steepness of the dependence in question (usually the value of $\alpha$ varies in a diapason from 1 to 3 - see, e.g., Newman [13]). Such 'rank form' of presentation is usually named Zipf's law. Naturally, when turning to logarithmic coordinates, we come to linear dependence with slope $-\alpha$. An example of such dependence is presented by Figure 3 - rank distribution of military expenses of 12 leading countries of NATO in 2018: USA, France, Great Britain, Germany, Italy, Canada, Turkey, Spain, Poland, Netherlands, Norway, and Greece. [The choice of such non-biological material is intentional: I wished to demonstrate rather broad diapason of applications of the statistical regularity discussed; official data were used, borrowed from Wikipedia.] The first rank undoubtedly belongs to USA (649 billion dollars), followed by France (63.8 billion), Great Britain (50.0 billion), and so on. Linear dependence (when using logarithmic coordinates for both axes) is evident; appropriate linear approximation for all 29 countries of NATO shows very high coefficient of determination $\mathrm{R}^{2}=0.96$ and the slope $\alpha=1.71$. (It is higher than the slope $\alpha=1.14$ for the entire world system - see Petrov, 2018, - which allows to propose that the structure of NATO has more rational features of organization than self-organized global system.) (Figure 3).

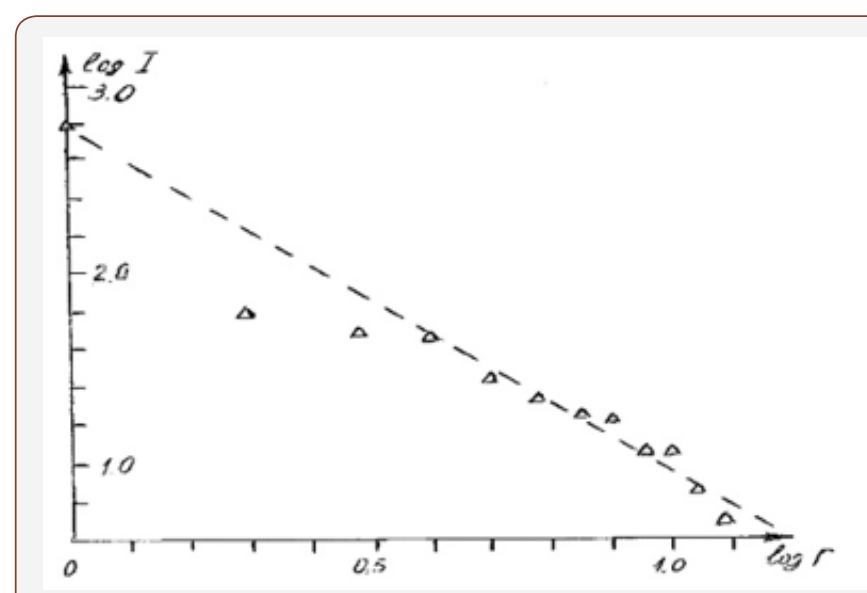

Figure 3: Distribution of 12 leading countries of NATO, over their ranks on defense budget, in logarithmic scales by both axes: USA, France, Great Britain, Germany, Italy, Canada, Turkey, Spain, Poland, Netherlands, Norway, Greece.

A little later we return to our main problem: impact of such distributions in the dynamics of the systems. But now what is interesting for us - which reasons are capable of causing the difference between such distributions and 'habitual' (for most researchers) ones, e.g., normal distributions - Gaussian? Besides, sometimes in reality, there is no strict border between these two types of distributions: the Power Law looks simply like a 'tail' of Gaussian distribution. Meanwhile, certain principal distinction does exist. At first sight, the main distinction is only the rarity of objects involved in the sample examined. Really, for instance, when investigating 'ordinary' students, we observe, as a rule, their 'normal' distribution over their achievements (exam marks and/ or other indicators), - whereas Nobel winners are subdued to the Power Law. The roots of these two kinds of results are different: in order to get good mark while exam, a student can EITHER possess brilliant abilities, OR hire a good coach, OR put on a mini-skirt (if there is a situation of a young lady and also young professor), OR spend much time for preparations, OR... - whereas to become a Nobel winner, a person should be BOTH talented, AND belong to a definite scientific school, AND be lucky when choosing country for his work, AND... In a word, in most cases we deal with two different principles: OR - and, on the contrary, AND, i.e., a certain kind of 'absolute harmony.' (Apropos, once upon a time, Gaussian distribution was theoretically deduced exactly proceeding from the assumption of very large number of impacts!).

Lyrical digression number three ('alcoholic'). Many years ago, when my colleagues and me were young and started to apply hyperbolic distributions in sociological measurements, in our circle a toast appeared, based on conditions of Zipf's law (and other close statistical regularities). At that time we were full of hopes, so this toast was devoted to the above principle 'AND': we wished for each of us - BOTH high scientific achievements, AND high social prestige, AND high salary, AND beautiful wives, AND luxurious house, AND... Only some of these 'Zipfean hopes' were realized because of social reasons! In general, hyperbolic distributions seem 
to be rather specific kind of statistical regularities: they belong to a class of 'stable non-Gaussian distributions,' in which, for instance, the calculation of average value occurs to be incorrect; some other peculiarities are also inherent to this class (see, e.g., [11,12]). However, now for us important are the dynamic sequences of such distributions.

The heart of the matter is in that as a rule, the top of such distribution 'decide' for the whole entity: the 'weight' of several top ranks - or perhaps the 'weight' of the first object (possessing $r=1$ ) sometimes exceeds $50 \%$ of the total sum; hence, this top is capable of determining the direction of the system's development. [Thus, namely such was the distribution of 'musical glory' in several national schools in Europe - see Kulichkin [14]. (The 'degree of glory' was ascribed to each composer by means of traditional measurement procedure: in dependence of the number of lines, devoted to his creativity in several musical encyclopedias.) It occurred, for instance, that at epoch of Mozart - personally he was the 'owner' of more than $50 \%$ of the total glory of German-Austrian musical school. Quite analogous was the situation in the epoch of Beethoven.] So, the top-persons can control the entire life of certain systems subdued to hyperbolic distributions.

The picture is aggravated by the phenomenon of centralization, which is typical accompaniment of the Zipf's law. As it was shown (see, e.g., Golitsyn \& Petrov [2]), the development of most multielement systems comes sooner or later to a certain centralized state where the elements are connected with each other not directly but via a certain core element. The most ocular demonstration is the evolution of telephone nets. In fact, at the early stages of their development, subscribes were connected with each other directly. However, the number of inter-element links $\mathrm{N}$ was rapidly growing together with the number of subscribes $n$ as the number of pairs among n elements: $N=n(n-1) / 2$. The solution of the problem turned out to be easy: the central telephone exchange was introduced within each net, where each subscriber was connected with other subscribers only through the exchange. The number of links sharply diminished to $\mathrm{N}=\mathrm{n}$.

Similar centralizing process was observed in the system of commerce, which evolved towards introducing one central currency element (commonly, it was gold), instead of the direct barter exchange of one product for another. Analogous centralizing evolution is typical for most systems in physics (which can be illustrated with a path towards deducing all the regularities proceeding from the only principle of optimality; apropos, exactly such is our systemic-informational approach), in religion, in the structure of the nervous system, and so forth. Due to the phenomenon of centralization, the role of the top-objects (or subjects) sharply increases - and sometimes it may occur dangerous for the very existence of the systems (both biological and social) - because of possible voluntary behavior of their tops. That is why the contemporary situation in the world seems to be extremely dangerous, being subdued to wills of quite small tops of social structures!
However, while this menace is not yet realized, we have enough time to turn to the second statistical regularity - which is the basis for stable functioning of any multi-generation system. Appropriate statistical law is also widely known: it is the phenomenon of 'regression to the mean.' Here our impact is zero (however, we proved this regularity empirically - see Petrov, Mazhul, \& Kulichkin [15]); simply it would be necessary to mention this classical regularity, briefly describing its evolutionary role. Once upon a time, some empirical confirmations of this regularity were observed by F. Galton [16]: he measured the height of sons of the tallest English fathers. These sons occurred - as a rule - not so tall, as their ancestors, - and this fact the researcher used for the conclusion of the 'regress' of English nation. Of course, such a conclusion was an error caused by rather specific character of the sample used: it dealt with the very 'top' of the distribution, i.e., the tallest fathers. Naturally, their sons should be not so tall - because their height depends not only of their hereditary features, but also of some other circumstances, mainly occasional ones. So, the above observations were the results of purely mathematical, statistical effect.

Meanwhile, various other analogous observations are still widespread in many fields, e.g., an opinion that "Nature relaxes on genuises' children" (i.e., sons are not so great; this phenomenon being known under different names - see Campbell \& Stanley [17]. In general, the dynamics of any constituent of a certain multielement system, can be influenced by two kinds of conditions:

a) the element's determination of its internal properties; in case of systems containing human beings, these 'influential variables' should include certain genetic features of subjects, as well as some results of the subject's previous (long-range) behavior, e.g., the subject's psychological attitudes, his/her level of knowledge, etc.

b) outer stochastic circumstances capable of changing the subject's behavior and/or parameters of mentality.

For instance, a student's behavior while an exam, can depend both on his/her internal abilities (or efforts spent by him/her for preparations) - and such occasional reasons as the student's headache, angry (or hungry) professor, bad weather, and so forth. Hence, we can suppose two possible 'polar' versions:

- When internal properties of the student (i.e., his/her own features) are much more influential than any stochastic circumstances. Let's consider a group of students which passed through exams after the first semester, so we can build their ordering (ranking) over their marks concerning a certain discipline. Later, after the next semester, we can again build their ordering (over their marks on this subject), and the ranking would occur tightly correlated with the previous one, because those students which occurred successful while the first half of the year, continue (due to their internal features) to be advanced also during the second half. So, the correlation between these hierarchies should be high. 
- When stochastic motives are prevailing. In such a case, the students' ranking over the marks after the second semester, would have nothing in common with their ranking after the first semester. Hence, the correlation between the hierarchies should be zero.

[The last version is typical also for systems belonging to nonliving matter - when the elements of such systems are devoid of any own (individual) properties. Thus, in the system of gaseous molecules (which are quite identical, possessing no 'personal properties,' except their velocities), the situation is constantly changing: the velocity of each molecule changes many millions times during each second; at each moment, the state of the molecule depends mainly on its previous collision - with another molecule, possessing unpredictable velocity. Hence, the evolution of the molecules' velocities is chaotic, as well as the evolution of their rankings over the velocities.]

In application to human accomplishments, interesting observations were realized by Hans Eysenck [18]: he studied the level of the index IQ of parents and their children. It occurred that usually "children of very dull or very bright parents regress to the mean, as do rather less strongly the children of dull and bright parents. Variation is maintained by children of average parents, some of whom are bright or dull, with a few very bright or very dull. This regression to the mean is largely responsible for the social mobility which is also characteristic of our society. <...> Not only intelligence, but also all other genetic variables contributing to genius or eminence, such as creativity, motivation and persistence would regress to the mean, leaving a much less distinguished progeny, on the average." This phenomenon is schematically illustrated with Figure 4.

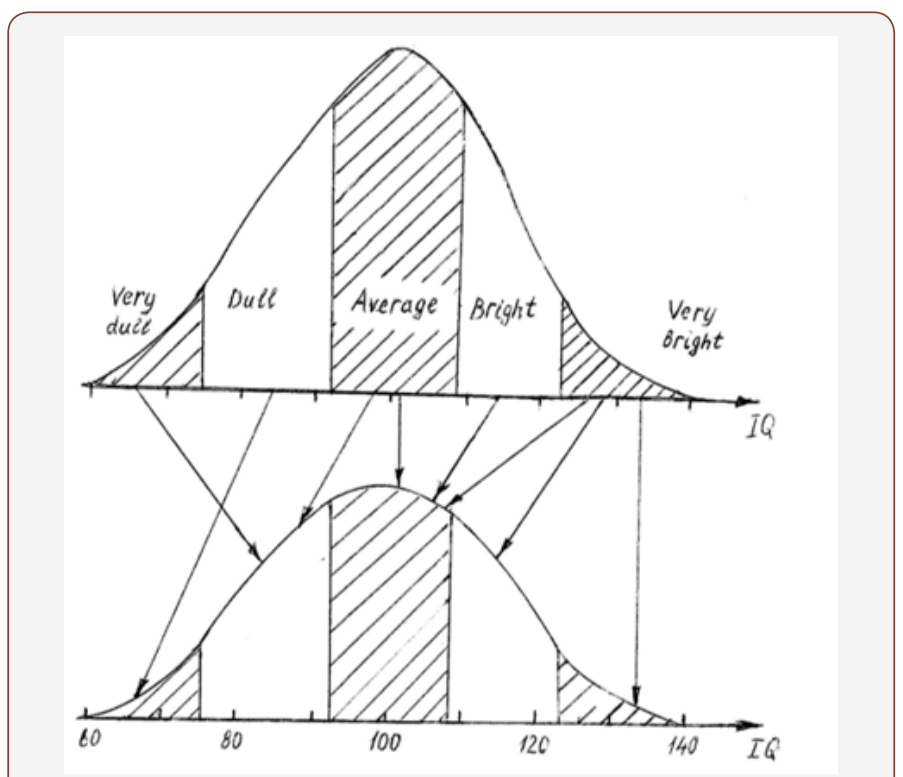

Figure 4: The phenomenon of 'regression to the mean': two points of the evolution (Eysenck's model).

Evidently, this phenomenon is so universal that it would be observed in different spheres. Recently we investigated this phenomenon on the material of subjects' "feeling of well-being" measured on Italian students (Lombardo, Mazhul, \& Petrov [19]): those persons which firstly were the most 'happy,' became - after a year and a half - not so happy, whereas the most 'unhappy' students became happier. Lyrical digression number four (about the fate of the population). When I was young, I was deeply impressed by an example concerning general systemic (cultural, evolutionary) sense of the phenomenon in question. Regression to the mean is capable of causing 'reverse' course of any system. In application to social systems, it is nothing else than the 'anti-utopian' logic of the socalled "abneguism" - phenomenon described in the brilliant short story "Null-P." written by physicist William Tenn (1920-2010). In this story (1951) a certain hypothetical situation is presented: one day the citizens of the U.S.A. decided that their President should possess 'average parameters' (over all the features, both physical and mental), the name of this first national leader was "Abnego." Afterwards such 'averaging criterion' was applied to all social positions, then this custom became widespread all over the world, and this phenomenon caused the total decay not only of creative abilities, - but also of the entire Mankind - 'reverse course of the history'. [Apropos, perhaps the death of some biological species was caused by analogous systemic reasons, though appropriate concrete mechanisms remain unknown for palaeontologists?] In general, the phenomenon discussed, 'brakes' the dynamics of the system. So, the balance of these two regularities: hyperbolic distribution and regression to the mean - comes to real development of various systems.

\section{Partial models: some 'inventions' of the evolution}

Turning to the course of the evolution, it seems reasonable to make ordering (though, perhaps, not very strict) of all models - in accordance with scales of their usage.

The most common (i.e., applied to rather broad circle of objects) are models of optimal information processing. They can be divided into two groups.

a. Models dealing with opposite ('polar') types of informational procedures within the above mentioned hierarchical structure (Figure 2). Here hidden are two possible versions of working with information (deduced by Maslov, see also Petrov, [20,21]):

- small portions of information are consequently processed at each given level of the hierarchical tower, with high precision, though rather slowly; as a result of such activity, 'selected' portions (relating to the most important part of the information processed) occur ready to be transmitted upwards, to the next level; as well, the criteria of selection are constantly formulated, renewed, and descended at the preceding level;

- large portions are transmitted upwards, to the next level, with high speed, however possibly with low exactness.

In total, this model is quite similar to our one (see Figure 2). In the framework of both models, the informational procedures remind of functioning of a certain bureaucratic organization (e.g., 
ministry) where the information concerning 'lower' objects (or subjects) is consequently reduced when ascending to the next, higher chief (e.g., Head of Department, Vice-Minister, and Minister). In application to human being, these two types of information processing - relate to dominating activity either of left or right hemisphere of the brain, respectively. In case of social processes, we observe periodical alternation of such 'left' and 'right' processes in the socio-political 'climate,' style of architecture, music, and painting - with full duration of cycles about 48-50 years (i.e., each 'left' creative generation dominates during 24-25 years, and then it is followed by 'right' generation - see, e.g., Petrov, 2001, 2008a), and dispersion about 5-6 years.

b. Another group of models deals mainly with keeping information in various forms, i.e., using either 'gestalt' objects or events perceived (meaning their images) - or sets of certain features (such as loudness, brightness, color, and so forth). The latter modality occurs to be much more advantageous. [In fact, going slightly ahead of our narration, let's suppose the situation, when it is needed to keep the information about some objects perceived in the subject's operative memory containing only 8 cells. Evidently, if to deal with gestalt images, it is possible to keep only 8 objects, whereas turning to two-gradation features, we come to $2^{8}=256$ objects!] So, namely this modality would dominate in our consideration. Here the key problem is to choose optimal number of gradations (needed to keep information) for each feature, and various informational models occur unanimous.

Thus, a model for 'non-motivated classifications' derived by Sukhotin [10], prescribes the most economic functioning of the system (i.e., requiring minimal resource expense) when each feature possesses $x=2$ or 3 gradations. As well, Fomin [22] built a model of analogous 'memory system' possessing W symbols and keeping information about objects, using combinations of their parameters, each containing $\mathrm{x}$ gradations. The capacity of such memory is $\mathrm{x}^{\mathrm{W} / \mathrm{x}}$; as far as the number of gradations should be whole, its optimal value would be again either 2 or 3. Finally, Golitsyn (see Golitsyn \& Petrov [2]) considered reaction of a system to outer stimuli, this reaction consisting of $\mathrm{N}$ independent components - 'degrees of freedom,' each component possessing $\mathrm{x}$ gradations with equal probabilities to be met. In the process of functioning, the system spends certain resource $\mathrm{R}$, the role of which can be played, in particular, by the efforts needed to keep information I about all the gradations. We receive maximal effectiveness of the system's reactions when $\mathrm{I}=\mathrm{R}$ $\ln \mathrm{x} / \mathrm{ax}$, i.e., when $\mathrm{x}=\mathrm{e}=2.71$ (again either 2 or 3 , a being a constant). And exactly such situations are met very frequently!

Models of tools providing optimal information storage. Here also two groups of models can be found, relating to two tasks (partly interwoven with previous ones)

a. Models aimed at optimal structure of memory (its 'hardware'). As far as our systemic-informational approach came to hierarchical structure of information processing, it needs three types of memory tools (characterized by different requirements concerning the functioning of our hierarchy):

- $\quad$ short-term, operative memory which deals with receiving primary information; here the main requirement is high speed of working (irrespective of capacity of appropriate tool); earlier it was shown that ternary divisions and ternary structures are preferable as the most economic; so, for this step we should use three channels of information transmitting, each channel transmitting binary signals, hence, the volume (capacity) of this tool $\mathrm{V}=2^{3}=8$ cells - units to be stored; this value is close to famous Miller's 'magic number seven, plus or minus two';

- $\quad$ long-term memory dealing with information processing within the given level; here the criteria of selection are formulated, which do not require high speed - but need large volume (without any temporal limitations);

- intermediate memory occupied by information transmitting to the next level; this tool is characterized by moderate requirements concerning both speed and volume.

Exactly these three types of memory were fixed empirically in numerous investigations.

b. Models dealing with algorithms ('software') inherent to the memory tools. Evidently, here the main requirement should be universality of the rules used, in order to provide high reliability of the algorithms proposed. Among appropriate 'inventions' of biological evolution we find such psychophysical phenomena as

$>$ The threshold of perception (subdued to well known principle 'all or nothing'); the value of the threshold would respond to devastating one cell out of the total capacity of the volume of operative memory $\mathrm{V}$ (which is 8 units) - or, on the contrary, to fill in additional empty cell; in any case, the value of relative threshold should be about 1/V, i.e., around .125 - irrespective of concrete nature of stimuli used (be it loudness, brightness, etc.), - and namely such values were empirically observed in numerous experiments;

$>\quad$ the perception of slight changes of signals; in order to provide surely such changes near the signal's intensity $F$ in rather wide range (taking into account that maximal intensity of sunlight exceeds the candle's light more than $10^{5}$ times), - it is necessary that the threshold of perception $\Delta$ would follow logarithmic law: $\Delta=\mathrm{c} \log \mathrm{F}$ (c being a constant), which is known as the so-called WeberFechner Law (see, e.g., Zabrodin \& Lebedev, 1977);

on the contrary, to perceive large changes of signals (also in a wide range of intensities), it is necessary to provide the reaction $R=b F^{\beta}$ (b being a constant), coefficient $\beta$ 
reflecting the steepness of the dependence, i.e., the importance of the given kind of stimuli - for the subject (usually the value of this coefficient varies - for different kinds of stimuli - in the range from 1 to 3 ); this theoretical regularity was also confirmed empirically, being known as the so-called Stevens' Law (see Zabrodin \& Lebedev [23]); besides, our calculations for such stimuli as the amount of information carried by objects' forms, evidence in favor of their rather high importance $(\beta=20)$.

These 'instrumental' models may be supplemented by other similar ones, relating to peculiarities of certain concrete situations in which the informational structures function. Thus, a partial model was construed for color perception (Golitsyn \& Petrov, 2005a): how many types of spectral detectors (each possessing bell-like distribution of spectral photo-response) are needed in order to identify the color of an object - under conditions of changing solar illumination? It occurred that optimal are three types, so it is not without reason that exactly such version (with maximums in green, yellow, and red colors) became the final point of the evolution of visual structures. (Four-types version a trial of which was once upon a time proved by the evolution on some birds in South America, was rejected by the evolution - as superflous!) Several other partial informational models were created both for 'hardware' and 'software,' to provide the system's progress while biological evolution, as well as its continuation, i.e., social and cultural development.

Till now we considered certain 'abstract' subjects (or even certain abstract 'multi-element systems'), almost without deepening into possible variety of the subjects' individual peculiarities. Meanwhile, these may occur decisive in some situations. That is why the next class of our informational models would be

\section{Models dealing with different individual types of information processing. To classify them, recently a hierarchical system was derived, based on five contrapositions, or 'binary oppositions' (see Petrov [24]:}

* Spatial splitting of the system's elements - into two halves, one of which serves conservation (keeping) the system as such - providing its identity, - whereas members belonging to another half, provide due mutability of the system's properties. In biological systems, exactly such is informational 'specialization' of females and males, respectively (about appropriate statistical distributions of the parameters of elements belonging to both halves, see in Geodakyan [25]).

* The above mentioned contraposition of two types of data processing Maslov [20], revealing itself in lefthemispheric or right-hemispheric dominance.

* The typology of human behavior proceeding from the subject's maximal value of the resource mobilization, meaning both the force of nervous processes and usual physical force Golitsyn [26]). Here two 'polar' types occur possible: weak and strong (known from the times of Hippocrates). As a result, the change of environmental conditions may cause quite opposite reactions: for 'strong' subjects, the increasing of the environmental entropy causes the growth of activity and its effectiveness, whereas 'weak' subjects show decreasing diversity of activity and its diminishing effectiveness (about appropriate measurements see, e.g., Mazhul et. al. [27]).

* The contraposition of introvertive and extravertive styles of thinking and behavior This well known phenomenon (see, e.g., some of its examples in political life of different countries - Simonton, 1994) is rooted in subject's orientation primarily either on internal or external source of the information subdued to processing by the system (e.g., personality), this orientation being provided by different neuromediators for these types.

* Finally, the degree of self-consistency (i.e., systemic character) of the features - both the above four parameters, and possible other ones, - characterizing the subject's mentality. It means statistical links, i.e., correlation between the parameters. To realize such correlation, needed are due neural mechanisms, e.g., statistical link between the hemisphericity - and the extraversion or introversion - which may be provided by appropriate hormonal means.

But it is time to turn to phenomena which are usually ascribed to the sphere of Culture - in the broad meaning of this term - e.g., according to Yury Lotman, who defined Culture as the totality of non-genetic information gathered, stored and processed by various human collectives. Here we will find a diversity of informational models relating to various kinds of human behavior.

As far as speech is one of the most important distinctive features of human beings, we should mention at least two groups of informational models

a) Models for perception of a speech flux. Here the most advanced is the so-called 'model of flickering mind,' containing three mechanisms: associative, correlative, and grammatical, which are switched on by turns, forming the sense of each fragment of the flux (see in detail: Golitsyn \& Petrov, Golitsyn \& Petrov, $[2,28]$ ). In order to support effective joint functioning of these three mechanisms, it is desirable to use certain 'special tools'; and really, one of such tools was invented by culture: it is nothing else than poetry (see also below; about empirical verification of appropriate effect, see, e.g., Golitsyn \& Petrov) [2].

b) Models for choosing the best channel for communication (usually acoustic one), finding optimal sound structure (meaning phonemic nomenclature and syllables), and reconstruction of the trajectory of its development (Golitsyn \& Petrov) [2]. 
Also important are informational models of principal perceptual parameters of our everyday life (see in detail: Petrov) [29].

- Three-dimensional model of Perceptual Space was built proceeding from the above mentioned fundamental tendency of expansion (see Section 1).

- Model of Perceptual Time was created proceeding from another fundamental tendency - of idealization. Functioning of both perceptual parameters presuppose to be supported by due cultural means, e.g., by means of music and poetry. Hence, our next group of informational models should deal with Models of the aesthetic sphere and its constituents: music, painting, poetry, women's beauty, prose, the phenomenon of humor, and so forth (see, e.g., Golitsyn \& Petrov [2,30,31]). As well, derived were numerous models dealing with optimal structure of devices used by art - and optimal frequency of their usage. However, here we intrude in the sphere of art studies.

\section{About vertical dimension and its statistical indicators}

Meanwhile, we are not sure that the above evolutionary innovations provide progressive development of the biological sphere. That is why it would be desirable to dwell upon the general problem of progress and 'vertical dimension' ('High' and 'Low') in the framework of the systemic-informational approach.

The empirical aspect of the problem of 'High' and 'Low' in the totality of different systems (physical, biological, social, cultural, etc.), was firstly put by German A. Golitsyn [32], who proposed the idea of the information as the main criterion of the 'height,' common both for science and the humanities. He mentioned that both physical world and mental one possess their own 'Bottom' and 'Top,' as well as the direction of movement (upwards) which can be named 'progress.' Moreover, he formulated a criterion characterizing the position of any system (or any element of the system) along this axis. This criterion is quantitative and purely formal, i.e., rather general and invariant over the nature of the system studied. It is nothing else than the degree of diversity, or the complexity, which is reflected by the entropy (or the 'own information' contained in the given system or its given element - let's remind of the above tendency A). That is why, for example, exactly those states (or elements) of the system should be esteemed as 'high' which can be met very rarely (see also Petrov) [33,34].

This criterion is rooted in the very long-range goal of the system's behavior: to increase its ability to survive, i.e., to keep its main structural features (to maintain its 'identity'). We see many examples of this phenomenon in physical systems (e.g., when considering the distribution of gaseous molecules in the vessel, over their velocities - see below), biological ones, social and cultural systems, and so forth. For instance, exactly those sociocultural systems possess the best long-range probability to survive, which are marked by large diversity of their elements, for example, political or cultural attitudes.
As for the elements of the systems, usually only rather rare phenomena are highly esteemed, treated as valuable, etc. (e.g., outstanding creative persons or genius works of art). As soon as such creative persons (or their works) can be met very seldom, they relate to large amount of information. So namely these elements should be considered as 'high' (see also Petrov \& Locher) [35]. However, the above considerations concerned only the criterion of necessity. So, it is impossible to use this criterion practically (e.g., when realizing measurements), without appropriate criterion of sufficiency, mainly because of a certain 'ambiguity.'

Really, in many cases not all the elements - or states of the system - which are rare (and hence, they are very informative), should be highly esteemed. For instance, some very stupid persons (as well as some too primitive works of art), are met rather seldom, so they respond to very large amount of information. Nevertheless, such phenomena are never highly esteemed (except some in humorous situations). Apropos, even in the sphere of non-living matter, we see quite analogous situations of 'ambiguity': for instance, Fig. 5 shows the distribution of molecules of hydrogen over their velocities - it possesses two zones of low-probable states (relating either to 'very slow' molecules or 'very fast' ones) (Figure 5).

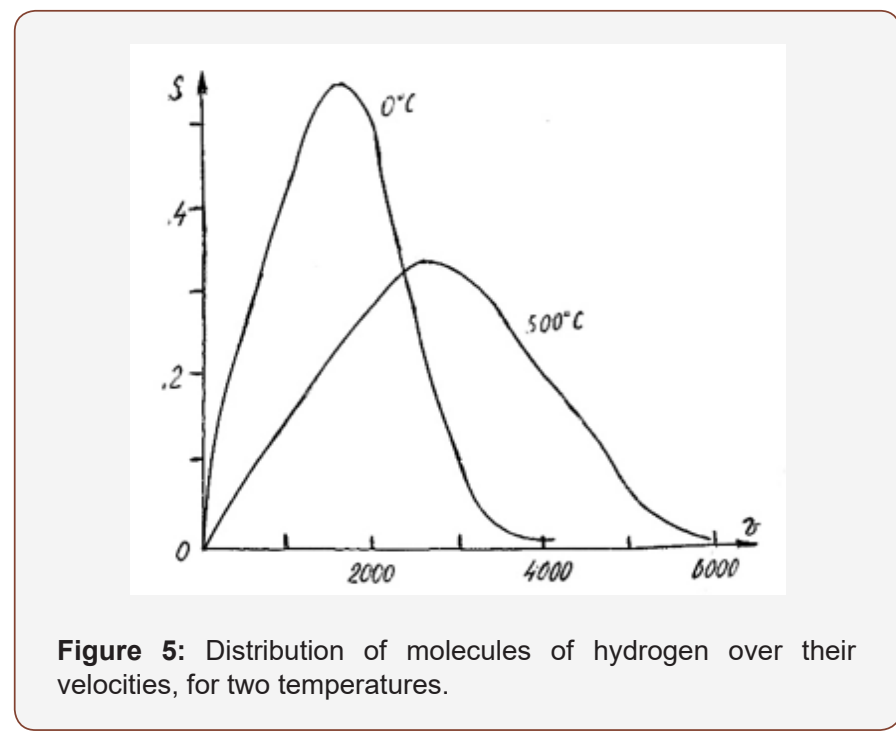

To overcome this difficulty (caused by the ambiguity) and to introduce genuine criterion of sufficiency, we should again return to the very foundations of the systemic-informational paradigm. In the framework of this paradigm, it occurs possible to obtain the criterion in question: its essence consists in the role of the elements considered (or states of the system studied) in the system's interactions with the environment - when changing the conditions of the system's life. Of course, exactly those elements (or those states of the system) should be treated as 'high,' which support the system's 'identity, in its struggle against possible destruction of the current (achieved) state of the system. Such situations are observed in the systems belonging both to living and non-living matter. For instance, in the above mentioned system of gaseous molecules, all of them participate in the struggle against the degradation of this system when transition from $500^{\circ} \mathrm{C}$ to $0^{\circ} \mathrm{C}$, but their impacts are different: the share of molecules possessing velocities about 3500 
$\mathrm{m} / \mathrm{sec}$, shows 9 times decreasing, whereas the share of molecules with velocities near $2000 \mathrm{~m} / \mathrm{sec}$, shows only 1.7 times decrease. Hence, the impacts of hot molecules are much more esteemed than the impacts of low-velocity molecules, so namely hot molecules should be evaluated as 'high.' Analogous distinction is inherent to the life of some advanced biological populations. Thus, the Evolution 'invented' different informational functions for males and females (see above): females are carriers of constant genetic information, whereas males carry genetic diversity needed for cases of unpredictable environmental changes. Hence, exactly males should be considered as more 'valuable,' 'high.' Finally, in contemporary social life, while wars, young ladies highly esteem a certain kind of men - officers, mainly because they play a great role in the defense of the country and therefore should be considered as 'high.' In general, certain 'vertical dimension' is inherent to all systems, from biology to sociology and cultural life. As for the sociocultural stage, some measurements were realized on the basis of the sociological investigation devoted to behavior while leisure: it occurred that most people engaged in 'low' professions (i.e., dealing with not-complicated labor), spend their time for contacts with 'low' kinds of art, whereas those engaged in 'high' labor activity, devote their leisure to 'high' art.

Appropriate indicators are really needed for social systems. In fact, how to clear up whether in the given moment, this country is moving towards progressive goal - or towards a decay? Very often, economic indicators are capable of generating deceptive impressions which afterwards result in the system's failure (sometimes catastrophic). To avoid such situations, recently special 'system of indicators of vertical growth' was derived (see in detail: Petrov) [36]. This system contains 22 statistical indicators, each of them relating to one of the above three fundamental tendencies (A, B, and C). For instance, one of statistical indicators dealing with 'resource tendency' (C), concerns such structural feature of the system, as the distribution of the resource among its elements in social systems it means the degree of social inequality. Here it seems reasonable to connect this distribution - with the amount of the total resource available. Appropriate model was derived in the framework of the systemic-information approach (Golitsyn) [1]. The model proceeds from some rather realistic presuppositions, the most substantial of which consists in that each element of the system (e.g., each person or each family) is capable of consuming certain resource in the range from zero to a certain upper limit responding to the total resource $\mathrm{E}_{\mathrm{l}}$. Then the distribution of elements over the levels of the resource consumption, responds to the entropy $\mathrm{H}=4\left(E_{1}-E^{2}\right)$, where $E$ is the total resource of the system. This is nothing else than the "parabolic dependence". In accordance with this formula, the entropy equals zero when $\mathrm{E}$ $=0$ and when $\mathrm{E}=\mathrm{E}_{\mathrm{p}}$, i.e., both when minimal and maximal resource available. This function possesses the only maximum, in the middle of the range - when $E=E_{1} / 2$. So optimal inequality in consumption (i.e., the most economic distribution of the resource consumed by the system's elements) should show the following evolution: from zero ("equality in poverty") - through maximal value ("inequality") - and again to zero ("equality in richness"). Such regularity is well known in economics as "Kuznets curve", named after Nobel winner Simon Kuznets.

That is why considering the direction of the evolution of any system in each given moment, it is necessary, first of all, to clear up which stage of the trajectory we are dealing with? Then it becomes possible to compare the direction of real dynamics observed with "due" direction of changes (this direction responding to the situation which is optimal for this evolutionary stage). In case of coinciding these two directions - this fact can be interpreted as an argument in favor of progressive dynamics, i.e., elevation of the system (its vertical growth). Otherwise, we observe the features of regressive evolution of the system at this moment. Nowadays all developed countries are in the 'last stage' of the evolution (going to the 'equality in wealth'). Hence, each of such countries should show growing homogeneity of income distribution - in case of progressive evolution. On the contrary, if a certain country demonstrates quite opposite tendency (i.e., growing social inequality), this fact should be an evidence of regressive evolution. As for the degree of social inequality, there exist various measures for this purpose, e.g., Gini coefficient.

Analogous indicators were derived proceeding from all three fundamental systemic tendencies $(A-C)$. Similar are the ways to judge about current direction of any system.

Apropos, some measurements were realized which dealt with such indicator as the 'test on subject's happiness' (based on visual illusions - see Mazhul \& Petrov, 2014; Petrov et al., 2015) [31,36]. Established was tight correlation between the degree of subject's feeling of well-being - and his/her position in the fields of career, health, and family life, and even the month of birth! So, 'full harmony' among various separate kinds of feelings does really exist! And perhaps, this harmony would be hopefully used practically, at least for scientific purposes? A little later we return to such possibility. But now our task is to clear up which tools were invented by the evolution, in order a member of the system (be him/her either rank and file - or outstanding person) could estimate his/her personal activity?

\section{Emotion as subjective indicator of current progressive / regressive movement}

Of course, the system's enhancement doesn't exhaust the entire nomenclature of possible dynamic processes. Each system can possess its own general inclination specific for each given moment of development. This relates, first of all, to situations when the behavior of the system is rather organized, i.e., more or less advanced systems. In such cases, there may appear a common direction of various behavioral acts which can be designated by the term 'goal,' as if the system 'knew the final point' of its activity. For instance, a certain prose-writer wishes to become a Nobel winner - it is his rather featured goal, and the overwhelming majority of his efforts are directed to create more and more perfect literary structures. On the contrary, another author dreams only of large payment, and for this goal, he writes only popular detective novels. Now in our consideration, there is no principal difference between 
the behavior of these two persons. As well, for us there is no difference between 'vertical estimations' of their goals; important is only the very fact of real existence of such goals.

Sometimes the goal of the system is 'reflected' neither by individual consciousness nor by social one. Moreover, the 'goal' can be ascribed even to the behavior of a non-living system - when speaking of its 'struggle for structural identity' (e.g., in the above example of the ensemble of gaseous molecules). Besides, there exist 'exotic situations' when the contradiction arises between the 'goal proclaimed' verbally - and real goal (i.e., behavioral one). Thus, in famous novel "1984" by Orwell, exactly such cases are described: efforts of special institutions proclaiming Peace, Abundance, and Love - are in reality directed towards War, Poverty, and Hatred, respectively. So, only real behavior of the system can be used in order to identify its current goal (if it does exist).

At the stage of the evolution when it came to definite individual organisms, their effective functioning can be supported by due informational means, i.e., indicators. And they do exist in the form of emotions. One of the most interesting approaches to the problem of emotions - 'biological theory of emotions' - is that of the famous Russian physiologist Pyotr K. Anokhin (1978) [37]. “He saw emotion as the criterion which makes it clear to an organism whether he moves towards a goal or away from it. In the first case emotion is positive ('pleasure'), in the second one - negative ('pain'). Thus pain and pleasure have an adaptive significance and help the organism to achieve its goals and to survive" (Golitsyn \& Petrov, 1995, p. 52) [2]. For instance, the above mentioned writer dreaming of the Nobel prize, evaluates each event of his literary career (e.g., a review in a literary journal), proceeding either from approaching to this goal (which generates his positive emotion) - or growing distance to it (negative emotion). As well, another writer dreaming mostly of great payment, is stimulated by emotions depending of the financial success of his novels.

In the framework of the systemic-informational approach, the dynamics of the emotion $\mathrm{E}$ when perceiving a certain new object, is determined by the information I received:

$E=d I / d t$. Figure 6 shows typical situation of such perception: dynamics both of the information and emotion (see also Golitsyn \& Petrov, 1995, pp. 51-55) [2]. This process consists of three principal phases: (Figure 6)

1. Rejection. Informational contact is not really established, so the system - in particular, the subject - cannot decode the signals from new object adequately, new information carries only further disordering, and the subject experiences decrease of the information. Hence, his emotion is negative. The subject can simply 'switch off' his attention and stop perceiving the object. But if he can compel himself to go on perceiving (i.e. to cross the threshold, about which we have written above) - sooner or later the next phase sets in.

2. Acceptance. The informational contact between the subject and the object perceived, becomes enough effective to provide growth of the information received (and processed). The emotion becomes positive, and the subject aspires to attract the object, to amplify the perceptive process and to prolong it.

3. Stagnation. The flux of the information from the object perceived, occurs exhausted, so it is almost nothing to be processed. That is why the emotion becomes negative again. The only means to break off this process, is to switch to any other, 'contrast' object. Then a new cycle of activity begins.

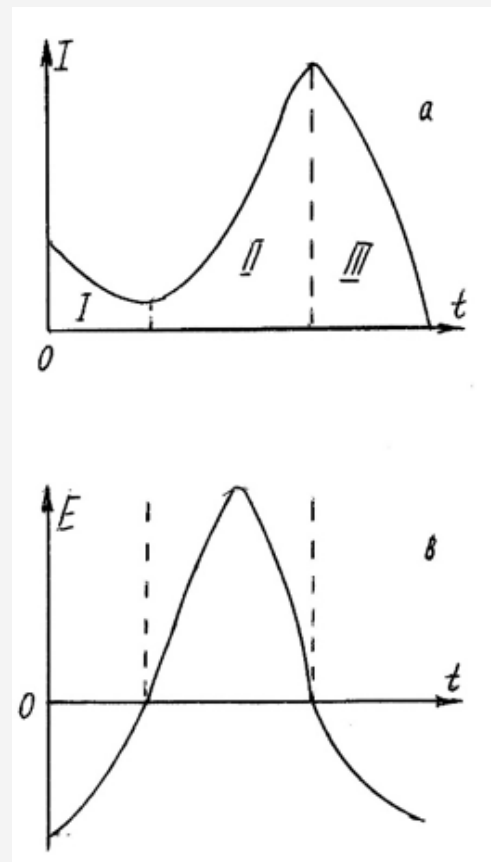

Figure 6: Dynamics of the information I and emotion $\mathrm{E}$ when perceiving new object.

There are many rather useful consequences of this model, which is universal and possesses very broad circle of applications. Thus, in the history of science, well known are 'three phases of the attitudes' to scientific innovations: firstly any new idea is resisted by most scholars (it is named 'mad' or 'absurd'), afterwards some of them occur to be interested in this innovation ('there is something valuable in it' - and it is approved), - but finally the innovation becomes trivial and dull ('who doesn't know this?'). Similar transformations are inherent to the fate of the directions of art. As well, recently this three-phase model permitted to deduce all the diversity of devices of art which can be used - and are really used - by music, painting, architecture, poetry, and prose (Golitsyn \& Petrov, 1995, pp. 143-176) [2]. [These devices are classified in dependence of the above phases of perception: each of them 'dictates' the means most suitable to minimize subject's negative emotion (i.e., to shorten the first phase of perception) and to maximize positive emotion (to prolong the second phase). Among the devices deduced, one can find such as rhyme, meter, rhythm, symmetry, contrast, nuance, golden section, vibrato, etc.] In general, the processes of 'selforganization' which occur possible due to this specific indicator - emotion, stimulate the subject's effective behavior, pushes him/ her not to spend time in vain. Due to this, the subject's chances 
to survive increase. Meanwhile, if the subject meets 'positively colored' objects regularly - during definite time interval - such 'local regularity' can be 'generalized', in the form of something like positive 'coloring' common for the whole interval. Further, if such events embrace several fields of the subject's activity - e.g., those connected with subject's health, social relations, and family life, it seems reasonable to introduce certain integral ('generalized') feeling of 'well-being,' or 'happiness.' Of course, quite symmetrical generalization can appear in cases of perceiving 'coinciding objects' - possessing common negative coloring, which occurs inherent to most fields of the subject's activity. In this situation we may tell that the subject feels him/herself 'unhappy.' Due to such 'generalized feeling,' the subject is 'pushed' (stimulated) to embrace, i.e., to aggregate many events in their totality (nor separately) - in order to conclude about the entire his/her 'life course' (at least during the given temporal interval): whether he/she is approaching to his/her goal. Such ascending to higher level of mental activity, may serve useful indicator for the subject's life course. As well, perhaps, this phenomenon was fixed in the above measurements of happiness?

\section{Half-hypothetical section: Harmony, intuition and forecasting}

Now we enter the final part of our narration which would seem to be rather specific - contrasting with the character of our previous text, as well as contradicting the logic of the overwhelming majority of other scientific texts of the author. The heart of the matter is the transition to hypothetical logic: now mainly indirect arguments will dominate the logic of the models (previously prevailing were either mathematical or strict empirical evidences). Lyrical digression number five (again methodological; hopefully, it would be the last digression in our narration). Sincerely, my soul of researcher is attracted namely by such hypothetical matters and indirect evidences - sometimes rather vague. [These are especially attractive when they occur many-sided: though each is indirect and vague, their totality may be very effective! It's a pity, there are no strict statistical methods for such situations.] And in general, the end of any strict scientific investigation would be followed by perspectives of its continuation, which are usually rather uncertain and vague! Moreover, sometimes I am frightened by the very fact of applying 'strict mathematics' when dealing with such delicate matters as intuition, sense of life, Zeitgeist, contraposition of High and Low, etc. Apropos, analogous fears were inherent even to the founder of the systemic-informational approach German A. Golitsyn (1937-1997) [1], who was my close friend: we wrote together and published (both in Russia and abroad) several joint books and many articles, and at the final stage of his scientific career, I fixed him up with work at my Institute (where I spent 36 years of my scientific life). So, German was absolutely sincere when speaking with me, e.g., about mathematical approaches to art studies: "As if a giant monster paws on a brittle tender body of art." Perhaps, these fears were his main motives (beside the censorship restrictions) not to publish his most valuable results (and these outstanding achievements became known only after his death). Nevertheless,
German was a pioneer in the field of mathematical psychology and quantitative art studies. He dared to 'tread on the throat of his own song' - and he became winner! That is why my soul is calm - though some traces of vagueness remained, meaning the preference for direct or indirect evidences, combined with hypothetical logic used.

The logic of our further narration will be mainly 'functional': we proceed from principal functions needed for the system's successful life and the tendencies revealed (including the results of our partial models described) - and we hope that appropriate mechanisms (realizing these functions) will be inevitably 'found automatically.' As if great Russian poet Ossip Mandelshtam wrote about such 'functional approach': Perhaps, before one's lips was born a whisper, In a pre-forest air were whirling leaves.

Our main hypothetical assumption is the need for certain special objects (or events) in mental life of each personality objects which would possess strong integrative abilities. Really, all the above considerations dealt mostly with separate kinds of activity; meanwhile, may appear certain special integrative means - the destination of which would be exactly integration of human's psychic life, i.e., the system of information processing. Previously we construed some partial models which dealt with phenomena serving for such integrative needs - but these were mostly 'byproduct functions' of the phenomena construed. Such 'partial phenomena' can be either 'natural,' i.e., construed by Nature, - or 'artificial' - created due to certain efforts of Culture (social system). For instance, among the letters we find a block of speech activity - together with appropriate integrative means assisting to joint functioning of all three semantic mechanisms involved in the subject's 'flickering perception' (see Section 3, models of group d3): associative, correlative, and grammatical. Here the main 'artificial means' is nothing else than poetry. [About empirical confirmation of such integrative impacts of poetical devices see: Golitsyn \& Petrov, 1995. [2]

Another rather effective kind of artificial integrative means (or perhaps, half-artificial - because they are rooted in Nature) is the phenomenon of women's beauty: though it proceeds from the natural need for the species' splitting into two halves each possessing its own informational specialization (see Section 3, class c), namely Culture is responsible for the concrete filling of this phenomenon. Due to its impact, the recipient occurs capable of experiencing 'harmony' relating to co-ordination (mutual agreement) of various features, some of them being of 'corporeal' origin, and some of 'mental' one. As well, when we dealt with such specific 'block' as spatial perception (see again Section 3, e), - our main requirement was to aggregate various images of objects - in the framework of the entire picture of three-dimensional Perceptual Everyday Space. [In such models, the picture of spatial world was formed under pressure of our fundamental tendency C - economy of resource.] And the model for our temporal representation - the so-called Perceptual Everyday Time - also aggregates (see again Section 3) various impressions connected with sequence of events perceived, their causal relations, and so on. [This concept is formed 
under pressure of limited capacity of human short-term memory.] Of course, both everyday concepts are supported by appropriate cultural means. Finally, exactly integrative function is the basis for the model of humor (Petrov, 2015) [31].

So, in each case, various 'sprouts' are observed - towards integrative inclination, as well as certain 'mental shifts' towards harmony. That is why we should suspect that the above both kinds of inclination would be involved in the general model of the subject's information processing - as well as cultural means providing its optimal functioning. Hence, undoubtedly the need for special 'integrative objects' (and/or events) does really should exist, being based on the subject's feeling of harmony when perceiving such objects. What might be the peculiarities of such special objects (events)? First of all, in the framework of appropriate model, we would provide certain 'peculiar status' of such special integrative objects (events): each of them should be exuded against the background of all other, 'habitual' objects (events). [Otherwise, these special objects would be lost in the common boundless sea, and they would be not capable of serving 'patterns,' or 'tuning forks' for the entire perception.] To provide such specific reaction, appropriate 'accompanying emotion' should be either extremely positive or extremely negative: in both versions the peculiar status discussed would be achieved. However, the version providing positive reaction, seems to be preferable - because positive emotion takes place when rather stable second phase of dynamics (see Fig. 6), whereas negative emotion relates to less stable first or third phases (depending on object's emergence or its disappearance, respectively). That is why namely 'extremely featured harmony' (which generates strong positive emotion) would be used as a basis for such objects (events).

[Nevertheless, sometimes 'extreme ugliness' can be also used. For instance, such a precedent is known: according Efroimsson [38], while pre-human and early human stages of the biological evolution, the feeling of females' beauty was formed as a result of rejecting extremely ugly subjects of the opposite sex. In general, when speaking of carriers of the information concerning our special integrative phenomena, - it would be better if they were young pretty ladies; exactly such are the so-called 'witches' - folklore personages in various cultures, - though sometimes we deal with ugly old women.] About the 'harmony' contained in our special integrative objects - there may exist the whole spectrum of kinds of 'synergy,' out of which the most 'influential' would be certain inter-level resonance, or agreement of results relating to different levels of the hierarchical structure of information processing. But to what temporal diapason should relate such agreement? Naturally, if to treat in such a manner the results describing current situation (nowadays one), - the fact of their agreement would be trivial, responding to no rarity. Quite opposite would be situations relating either to the past or, preferably, to the future, i.e., to forecasting. Really, in future the diapason of possible events is giant, so the probability of random coinciding is extremely low, such case being perceived as a miracle. So, existence of definite miracles responds to the needs of the system's optimal information processing. And in particular, among such miracles we see the phenomenon of witches, which is inherent to any cultural system.

Nevertheless, we see different social attitudes to this phenomenon. Thus, in medieval epoch in most West-European countries, the witches were disclosed and burnt. [In one Austrian castle, I saw even special luxurious hall for burning witches, where several hundreds of them ended their days.] On the contrary, in Russia traditional attitude to this phenomenon was even encouraging. So, perhaps, it was not without reason great Russian prose-writer Nikolay Gogol noticed - though in half a joke - that in his native Ukrainian settlement, all market-women were witches. Hence, they could survive all the changes! And the role of this phenomenon in different cultures requires separate investigation.

Meanwhile, undoubtedly, some features of personality should be inherent to carriers of this phenomenon. Moreover, as far as rarity would be necessary for its functioning, the above mentioned principle AND (see Section 2) should work, resulting in hyperbolic distributions. Hence, the next question arises: which namely features would occur prevailing? Evidently here we should consider those informational mechanisms through which the phenomenon of 'witch forecasts' can be realized. Most likely, any success can be provided by certain feedback connecting the person's activity with its various 'traces,' i.e., a set of consequences. Most of them would show - in the framework of the picture of the forecasted future - high mutual correlation, i.e., good agreement, harmony. If such harmony takes place - it is an evidence of the foreteller's good intuition. (Exactly such many-sidedness is the main peculiarity inherent to intuitive thinking.) However, rather developed intuition is typical for right-hemispheric mentality. Therefore, mostly righthemispheric subjects should possess such 'witch-like' features. A propos, it may be one of the reasons that mostly females were convicted - out of all possible personages. Besides, perhaps the right-hemispheric orientation of the entire Russian culture is also responsible for the encouraging attitude to witches?

Maybe, prospective are empirical investigations aimed at fixing months of birth of witches - because right-hemispheric style is typical mostly to persons born in Winter (see Marzullo, 1996) [39]. As well, prospective may occur empirical investigations establishing links between the phenomenon of witches - and various other features of personality (see Section 3). Moreover, when we deal with intuition and similar procedures of 'parallel processing' large portions of information - it seems reasonable to pay attention to analogous informational processes realized by octopuses? Each of them possesses 8 brains, functioning in parallel, and perhaps, it is not without reason they are ascribed to some kinds of 'magic forecasting'? But now we are on the borderline of the sphere of mystics. Or perhaps, it is time to construe something like General Theory of Miracles?

What is doubtless - now realized occurred the so-called "Euler's Program" (see in detail Levich, Golitsyn \& Levich, Petrov,) [40,41,6]at least in relation to the entire complex of the most complicated problems dealing with human mentality and culture. Once upon 
a time this Program was announced by genius mathematician Leonhard Euler (he was an Academician both of Berlin and Saint Petersburg Academies of Sciences). He proposed an idea of logical deducing all principal regularities of the Universe - proceeding from the only 'principle of optimality.' During more than two centuries, this ambitious task was considered to be unreal. Now the situation starts to change - and some fragments (e.g., relating to physics) of the new worldview appear. Hopefully this publication will contribute to solving this task [42-47].

\section{Acknowledgement}

The author wishes to express his deep gratitude to his late friends Dr. German GOLITSYN, Prof. Alexander LEVICH, and Prof. Colin MARTINDALE - for numerous fruitful discussions, without which this paper couldn't be realized.

\section{Conflict of Interest}

\section{No conflict of interest.}

\section{References}

1. Golitsyn GA (1997) Information and creation: On the road to integral culture. Moscow: Russky Mir (in Russian).

2. Golitsyn GA, Petrov VM (1995) Information and creation: Integrating the 'two cultures.' Basel; Boston; Berlin: Birkhauser Verlag.

3. Golitsyn GA, Petrov VM (2005) Social and cultural dynamics: Long-range tendencies (Informational approach). Moscow: KomKniga (in Russian).

4. Golitsyn GA, Petrov VM (2005a) Information and biological principles of optimality: Harmony and algebra of living. Moscow: KomKniga (in Russian).

5. Petrov VM (2007) The information approach to human sciences, especially aesthetics. In: C Martindale, P Locher, V Petrov (Eds.), Evolutionary and neurocognitive approaches to aesthetics, creativity, and the arts. Amityville, NY: Baywood Publishing Co, pp. 129-148.

6. Petrov VM (2017) Modeling worlds of life, culture, and art: Primary 'bricks' to be used in deductive construing the Mental Universe. Rivista di Psicologia dell'Arte 28: 7-42.

7. Petrov VM (2018) Statistical distribution and distribution of power: In search for harmony in international relations. Model Assisted Statistics and Applications 13(3): pp. 271-278.

8. Fano RM (1951) Transmission of information. New York; London: M.I.T. Press \& Wiley, USA.

9. Eigen M (1951) Self-organization of matter and the evolution of biological macromolecules. Berlin: Springer.

10. Sukhotin BV (1983) Classification and sense. In: VP Grigoryev (Ed.), Problems of structural linguistics 1981. Moscow: Nauka, Russia, pp. 5265 (in Russian).

11. Petrov VM, Yablonsky AI (1980) Mathematics and social processes: Hyperbolic distributions and their applications. Moscow: Znaniye (in Russian).

12. Petrov VM, Yablonsky AI (2013) Mathematics of social inequality: Hyperbolic distributions in studies of socio-cultural processes. Moscow: Librokom (in Russian).

13. Newman MEJ (2005) Power laws, Pareto distributions and Zipf's law. Contemporary Physics 46: pp. 323-351.

14. Kulichkin PA (2007) 'Evolutionary genius' and the intensity of artistic life: Who makes musical history? In: L.Dorfman, C.Martindale, V Petrov (Eds.), Aesthetics and innovation. Newcastle: Cambridge Scholars Publishing, pp. 363-396.

15. Petrov VM, Mazhul LA, Kulichkin PA (2017) 'Regression to the mean' in creative abilities: A trial to measure the pedagogic process. Proceedings of the 27th National Scientific Symposium with International Participation 'METROLOGY AND METROLOGY ASSURANCE 2017,' Sozopol, Bulgaria: 315-318.

16. Galton FG (1877) Typical laws of heredity. Nature 15: 492-495, 512-514, 532-533.

17. Campbell DT, Stanley JS (1976) Experimental and quasi-experimental design for research. Chicago: Rand McNally.

18. Eysenck H (1995) Genius. The natural history of creativity. Cambridge, UK: Cambridge University Press.

19. Lombardo G, Mazhul L, Petrov V (2016) Visual test on happiness revisited: Temporal long-range reproducibility. Integrative perspective in humanities, pp. 46-53.

20. Maslov S Yu (1983) Asymmetry of cognitive mechanisms and its consequences. Semiotika i informatika 20: 3-34 (in Russian).

21. Petrov VM (2008a) Social and cultural dynamics: Fast processes (Information approach). Saint Petersburg: Aleteya (in Russian).

22. Fomin SV (1964) Systems of counting. Moscow: Nauka (in Russian).

23. Zabrodin Yu M, Lebedev AN (1977) Psychophysiology and psychophysics. Moscow: Nauka (in Russian), Russia.

24. Petrov VM (2019) Dynamics of social and cultural systems: archetypical - though rather preliminary, half-hypothetical approach. Rivista di Psicologia dell'Arte, No. 30 (in press).

25. Geodakyan VA (1983) Evolutionary logic of sex differentiation and the longevity. Priroda 1: 70-80 (in Russian).

26. Golitsyn GA (2013) Emotional states: informational typology, regularities of formation, orientation of art. Quantitative methods in art studies. Proceedings of international conference in memory of German A. Golitsyn, September 20-22, 2012. Yekaterinburg: Artefakt: 45-49 (in Russian).

27. Mazhul LA, Petrov VM, Mazhul KM (2016) Correlation-based measurements: The concept of 'neural force' revisited. Proceedings of the 26th National Scientific Symposium with International Participation 'METROLOGY AND METROLOGY ASSURANCE 2016.' Sozopol, Bulgaria. pp. 292-298.

28. Golitsyn GA, Petrov VM (2007) Information. Behavior. Language. Creativity. Moscow: LKI Publishers, Russia. (in Russian).

29. Petrov VM (2008). Ternarity in thinking, culture, and art: Systemicinformational roots of subconsciousness. Psychology - Journal of Higher School of Economics, vol. 5, No. 4. Pp. 3-18 (in Russian).

30. Koptsik VA, Ryzhov VP, Petrov VM (2004) Etudes on the theory of art: Dialogue between science and the humanities. Moscow: OGI (in Russian).

31. Petrov VM (2015) From informational roots - to humorous fruits (A 'global approach' to very interesting phenomena - VIPs of our mental world), Rivista di Psicologia dell'Arte 26: 5-28.

32. Golitsyn GA (2000) 'High' art and 'Low' art: The systemic role of an elite subculture. Journal of Russian and East European Psychology 38: 28-44.

33. Petrov VM (2002) 'High' against 'Low': Hierarchies in Nature, Culture, and Art. Bulletin of Psychology and the Arts 3(1): 3-9.

34. Petrov VM (2004) Quantitative methods in art studies. Handbook for students. Moscow: Academic Project (in Russian).

35. Petrov VM, Locher PJ (2011) Genius: A son of his time or a Rara Avis? Empirical Studies of the Arts 29(1): 111-128.

36. Petrov VM (2014) Measuring social progress: Indicators of vertical growth. Key Engineering Materials 613: pp. 474-481.

37. Anokhin PK (1978) Beitrage zur allgemeinen Theorie des funktionalen Systems. Jena: Fischer.

38. Efroimsson VP (1995) Genetics of ethics and aesthetics. Saint Petersburg: Talisman (in Russian).

39. Marzullo G (1996) Month of birth, creativity, and the 'two classes' of men. New York: Per Aspera Books, USA. 
40. Levich AP (2010) Art and method in describing the dynamics of systems. Mir Psikhologii (World of Psychology) 3: pp. 185-203 (in Russian).

41. Golitsyn GA, Levich AP (2009) The principle of the information maximum and variational principles in sciences. In: VMPetrov, AV Kharuto (Eds.), Art studies and information theory, Moscow: KRASAND (in Russian), pp. 52-87.

42. Golitsyn GA, Petrov VM (1997) The principle of the information maximum, Zipf's law, and measurement of individual cultural development. In: L Dorfman, C Martindale, D Leontiev, G Cupchik, V Petrov, P Machotka (Eds.), Emotion, creativity, and art. In two volumes. Perm: Perm State Institute of Arts and Culture 1: pp. 179-221.

43. Martindale C (1990) The clockwork muse: The predictability of artistic change. New York: Basic Books, USA.
44. Mazhul LA, Petrov VM (2014) Harmony of existence, visual illusions, and test for subject's 'happiness.' Rivista di Psicologia dell'Arte 25: pp. 27-33.

45. Petrov VM (2001) Creativity in art: Stylistic waves and monotonic evolutionary trends (Information approach). Bulletin of Psychology and the Arts 2(1): pp. 30-33.

46. Petrov VM, Mazhul LA, Lombardo G (2015) Measurement of happiness - seasonal determination. Proceedings of the 25th National Scientific Symposium with International Participation 'METROLOGY AND METROLOGY ASSURANCE 2015,' Sozopol, Bulgaria: 373-377.

47. Simonton DK (1994) Greatness: who makes history and why? New York: The Guildford Press, USA. 